\title{
Wissen als Basis für Innovationen
}

\begin{abstract}
Das Wissenschaftsjahr 2012 - Zukunftsprojekt ERDE des Bundesministeriums für Bildung und Forschung (BMBF) steht im Zeichen der Forschung für nachhaltige Entwicklungen: Sie ist der Schlüssel für die Zukunft. Es gilt, einen Forschungsansatz zu schaffen, der wirtschaftliche, ökologische und soziale Aspekte gleichzeitig umfasst, ohne ein Problem auf Kosten eines anderen zu lösen. Von Wilfried Kraus
\end{abstract}

K limawandel, Bevölkerungswachstum oder Biodiversitätsverlust sind globale Herausforderungen, die in den nächsten Jahren zu meistern sind. Wissenschaftlerinnen und Wissenschaftler arbeiten nicht nur in der Umwelt- oder Energieforschung, sondern ebenso in den Wirtschafts- und Geisteswissenschaften daran, die Erde auch für kommende Generationen zu bewahren.

Das Wissenschaftsjahr 2012 - Zukunftsprojekt Erde widmet sich dieser Forschung für nachhaltige Entwicklungen. Das Thema des aktuellen Wissenschaftsjahres setzt damit die Tradition fort, Zukunftsthemen unserer Gesellschaft über Fachgrenzen hinweg zu diskutieren und Wissenschaft in den Dialog mit Gesellschaft und Wirtschaft zu bringen.

\section{Fragen, auf die es mehr als eine Antwort gibt}

Im Wissenschaftsjahr 2012 - Zukunftsprojekt Erde werden Fragen gestellt, auf die es mehr als eine richtige Antwort gibt: Wie wollen wir leben? Wie müssen wir wirtschaften? Und: Wie können wir unsere Umwelt bewahren?

Das Wissenschaftsjahr für nachhaltige Entwicklungen macht deutlich, wo die Leistung der Wissenschaft liegt: Sie hat den Mut, bisherige Lösungsstrategien zu hinterfragen. Sie beleuchtet ein Problem von allen Seiten. Ihre Forschungsergebnisse bieten die Basis für Politik und Gesellschaft, fundierte Entscheidungen zu treffen. Der Wirtschaft liefert sie Grundlagenwissen, Innovations- und Investitionspotenzial.

Das BMBF fördert im Sinne nachhaltiger Entwicklungen gegenwärtig mehr als 500 internationale Projekte in 62 Ländern auf fünf Kontinenten. Allein im Rahmenprogramm „Forschung für nachhaltige Entwicklungen“ (FONA) stellt das BMBF bis 2015 mehr als zwei Milliarden Euro Fördermittel für die Entwicklung nachhaltiger Innovationen bereit. FONA fördert dabei den gesamten Forschungsprozess von der Grundlage bis zur Anwendung und blickt insbesondere auch auf die ökonomische Dimension.

Der Förderschwerpunkt „Wirtschaftswissenschaften für Nachhaltigkeit" zielt darauf ab, volkswirtschaftliche Forschung zu Nachhaltigkeitsfragen anzuregen: Welche wirtschaftlichen, sozialen und ökologischen Leitplanken braucht die globalisierte Wirtschaft? Wo liegen aus ökonomischer Sicht heute und morgen die Ansatzpunkte für eine erfolgreiche Umweltpolitik? Wie können zielgerichtete Innovationen die Gesellschaft auf ihrem Weg zu mehr Nachhaltigkeit effizient unterstützen? Diesen Fragen widmen sich die beteiligten 18 Forschungsprojekte auf unterschiedlichste Weise.

\section{Beispielhafte Projekte}

So entwickelt das Projekt STROM des Instituts für Automobilwirtschaft und Industrielle Produktion der Technischen Universität Braunschweig „strategische
Optionen der Automobilindustrie für die Migration nachhaltiger Antriebstechnologien“. Am Lehrstuhl für Betriebswirtschaftslehre der Universität DuisburgEssen forschen Wissenschaftler im Projekt NaREM an einem „Marktdesign für nachhaltige regionale elektrische Energiemärkte“. Mit der Frage, wie Ökosystemdienstleistungen effizient und sowohl inter- als intragenerationell gerecht genutzt werden können, beschäftigt sich beispielsweise das Projekt des Departments Nachhaltigkeitswissenschaften der Leuphana Universität Lüneburg.

\section{Mehr als 100 Partner laden zum Mitmachen ein}

FONA ist einer der zentralen Kooperationspartner des Wissenschaftsjahres 2012 - Zukunftsprojekt Erde. Das vielseitige und hochkarätige Spektrum der Partner macht den besonderen Reiz des Wissenschaftsjahres für nachhaltige Entwicklungen aus. Mehrere Hundert Mitwirkende werden sich mit eigenen Informations- und Mitmachangeboten am Wissenschaftsjahr 2012 - Zukunftsprojekt Erde beteiligen: mit Ausstellungen, Wettbewerben, Vortragsreihen oder Diskussionsveranstaltungen.

Die Wissenschaftsjahre werden vom Bundesministerium für Bildung und Forschung (BMBF) gemeinsam mit der Initiative Wissenschaft im Dialog (WiD) sowie zahlreichen Partnern aus Wissenschaft, Wirtschaft, Politik und Kultur ausgerichtet.

\section{Weitere Informationen \\ www.zukunftsprojekt-erde.de}

I AUTOR + KONTAKT

Ministerialdirigent Wilfried Kraus ist Unterabteilungsleiter Nachhaltigkeit, Klima, Energie im Bundesministerium für Bildung und Forschung und Leiter der Projektgruppe Wissenschaftsjahr 2012 - Zukunftsprojekt Erde.

E-Mail: redaktionsbuero@zukunftsprojekt-erde.de 